\title{
Satiation treatment and learning of competitional and non-competitional pairs of a paired-associate task
}

ARJUN P. PUROHIT

QUEEN'S UNIVERSITY, KINGSTON, ONTARIO

\begin{abstract}
Satiation treatment to competing responses did not improve learning of the competitional pairs but the same treatment to response members of the non-competitional pairs resulted in decreased learning of non-competitional pairs of a paired-associate task. It is suggested that satiation treatment results in generation of reactive inhibition but does not effect the semantic structure of the satiated material.
\end{abstract}

\section{Problem}

Lambert \& Jakobovits (1960) have reported measurable decrements in the intensity of the semantic ratings of continuously repeated words. Using the same verbal repetition procedure with digits, they have demonstrated an increase in the latency of solution of computational tasks which involved satiated digits. More recently, Kanungo \& Lambert (1962) and Kanungo, Lambert, \& Mauer (1964) have shown a decrement in the learning of a paired-associate task which consisted of satiated words as response words. The findings give considerable support to the "semantic loss" theory of satiation effect, as proposed by Lambert \& Jakobovits (1960).

This study is designed to verify Kanungo et al's finding, and to test another prediction of the "semantic loss" theory: there will be significant improvement in learning of a competitional paired-associate task if the competing responses receive satiation treatment.

\section{Procedure}

The paired-associate learning task employed in this experiment consisted of four correct and four incorrect multiplications. The stimulus member of each multiplication pair consisted of two single-digit numbers with a notation of multiplication between them and a notation of equation after them. The response member of each "correct" pair was the correct two digit product of its respective stimulus member. The response members of the "incorrect" pairs, however, were two digit numbers which were not correct products of any of the stimulus members.

Prior to the learning session, the experimental group, consisting of 12 senior high-school students, was given satiation treatment on the correct products of all the eight stimulus members. The procedure was essentially similar to that used by Lambert \& Jakobovits (1960). Briefly, the S was asked to repeat each number aloud for $15 \mathrm{sec}$. (and as swiftly as possible) immediately following its enunciation by the
E. All the eight numbers were presented three times and in a randomized order. No satiation treatment was given to the control group, also consisting of 12 senior highschool students.

Both groups were then required to learn the list of multiplications by anticipation method, a Hull-type memory drum being used for presentation. The list was continuously presented as follows: stimulus 1 (2 sec.) and then stimulus 1 and response 1 (2 sec.); stimulus 2 ( $2 \mathrm{sec}$.$) and then stimulus 2$ and response 2 (2 sec.); and so forth. Each $\mathrm{S}$ learned the task individually and serial position effects were avoided by the use of five random orders of the list. Twenty-four trials were given after the initial familiarization trial.

\section{Results}

Figure 1 represents the learning curves of both groups on "correct" and "incorrect" pairs in terms of blocks of 4 trials. A 2 (groups) by 6 (blocks of trials) analysis of variance was made of the learning scores on the correct and the incorrect pairs in the task. As Fig. 1 suggests, (a) there was no difference between the two groups in their learning of the incorrect pairs, and (b) there was no difference between the two groups in the early stages of learning of the correct pairs, the experimental group showing poorer performance with the progress of the learning trials. Only Trial Blocks for incorrect pairs $(F=14.28 ; d f=5 / 110 ; p<.01)$

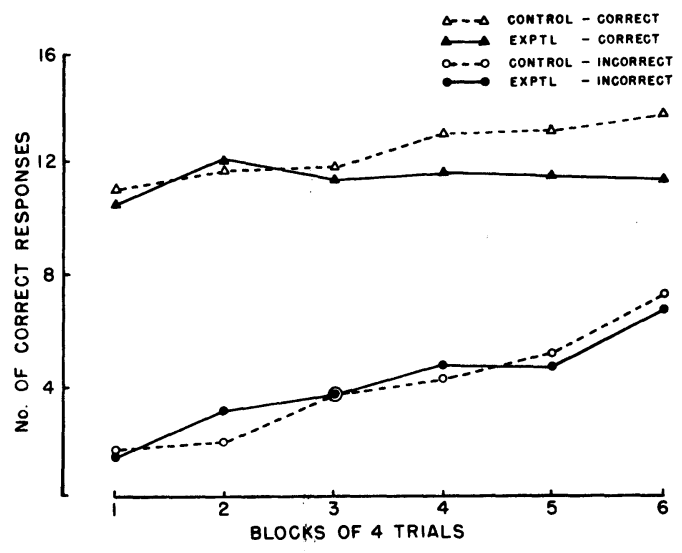

Fig. 1. Leaming curves of correct and incorrect pairs in control and experimental groups. 
and the Trial Blocks by Satiation Treatment $(F=4.48$, $\mathrm{df}=5 / 110 ; p<.05)$ were significant.

These results have important implications on the interpretation of "semantic loss" theory of satiation effect. The fact that satiation treatment of the competing responses did not improve learning in any stage in the case of incorrect pairs leads one to suspect that the satiation treatment did not reduce the semantic structure of the numbers. On the other hand, the satiation treatment did effect the learning of correct pairs adversely with the progress of learning trials. One plausible explanation might be that satiation treatment generated reactive inhibition, which was not sufficiently effective in the beginning but became so with additional reactive inhibition because of further experience with the satiated numbers. If this is so, one might conclude from these results that satiation treatment results in generation of reactive inhibition but does not effect the semantic structure of the satiated material.

\section{References}

Jakobovits, L. A., \& Lambert, W. E. Semantic satiation in an addition task. Canad. J. Psychol., 1962, 16, 112-119.

Kanungo, R., \& Lambert, W. E. Effects of variations in amount of verbal repetition on meaning and paired-associate learning. $J$. verbal Learn. verbal Behav., 1964, 3, 358-361.

Kanungo, R., Lambert, W. E., \& Mauer, S. M. Semantic satiation and paired-associate learning. J. exp. Psychol., 1962, 64, 600607.

Lambert, W. E., \& Jakobovits, L. A. Verbal satiation and changes in the intensity of meaning. $J$. exp. Psychol., 1960, 60, 376-383. Note

1. This research was supported by National Research Council of Canada. 